\title{
LPE-Like growth of YIG ferrimagnetic thin films by pulsed laser ablation with molten droplets
}

\author{
Akiharu Morimoto ${ }^{1}, Y_{0 s h i m i ~ M a e d a}{ }^{1}$, Toshiharu Minamikawa ${ }^{2}$, Yasuto Yonezawa $^{2}$, \\ and Tatsuo Shimizu ${ }^{1}$ \\ ${ }^{1}$ Department of Electrical and Computer Engineering, Kanazawa University, Kanazawa 920-8667, Japan, fax: +81- \\ 76-234-4900, amorimot@ec.t.kanazawa-u.ac.jp \\ ${ }^{2}$ Industrial Research Institute of Ishikawa, Kanazawa 920-0223, Japan
}

\begin{abstract}
Yttrium iron garnet (YIG) films were grown by pulsed laser ablation (PLA) on (111) Gadolinium Gallium Garnet (GGG) substrates. The second harmonic of YAG laser (532 $\mathrm{nm}$ ) with a high laser fluence was employed for producing YIG droplets efficiently. It was found that YIG films prepared at RT substrates have a large number of solidified droplets with various sizes. Highly oriented YIG crystals were grown on the (111) GGG substrate heated at $860{ }^{\circ} \mathrm{C}$ by PLA using a large number of molten droplets, suggesting an LPE-like growth. This YIG film shows a small ferrimagnetic resonance linewidth of 7.5 Oe. This value is quite small among films prepared by vapor-phase-epitaxy techniques.
\end{abstract}

PACS: $\quad$ 61.10.Nz, 68.55.-a, 76.50.+g 


\section{Introduction}

Pulsed laser ablation (PLA) process is known to have a widely spreading sizedistribution in ejected particles from atoms and molecules to droplets with micron size depending on the laser fluence and the environment $[1,2]$. Presence of droplets on the deposited films is one of the well-known drawbacks in the film deposition by PLA because of the poor surface morphology. So far, for obtaining a high quality film using PLA, it has been believed that the droplets must be eliminated $[3,4]$. The droplets in PLA are, however, thought to be a quite unique feature among the various vapor phase epitaxy (VPE) techniques from the viewpoint that the PLA process includes solidification through molten droplets from the targets. Principally, liquid phase epitaxy (LPE) is governed by an equilibrium process, while VPE is by a non-equilibrium process. In order to grow a high quality film with a low defect density, a crystal growth by PLA using a large number of molten droplets will be an attractive technique that allows LPE-like crystal growth via VPE-like technique.

Firstly, this idea was examined by using Ge. Ge is a material with a single element with a low melting temperature of $937^{\circ} \mathrm{C}$. This feature will easily allow us a crystal growth just below the melting point by using a conventional vacuum chamber without any problem on segregation or off-stoichiometry. The result revealed that an epitaxial growth of Ge film was attained on Si by PLA using a large number of molten droplets [5]. On the other hand, yttrium iron garnet (YIG) is known to be an excellent material for microwave applications, for instance, delay lines [6], filters [7], resonators [8], S/N-enhancers [9], etc. We presented the first report on the preparation of garnet films by PLA using ArF excimer laser [10]. So far, many YIG films have been, however, 
prepared mainly by LPE technique. For YIG application to various microwave devices, it is desirable to prepare the films by VPE-like technique, without direct soaking the device to the molten material with a high temperature. The present LPE-like deposition technique is expected to allow us to fabricate devices throughout in dry processes.

\section{Experimental procedure}

In this work, the second harmonic of YAG laser $(532 \mathrm{~nm})$ was employed for producing YIG droplets efficiently. Employed laser was a Q-switched Nd:YAG laser of Qauntel Int. Inc. (YG661-10). The laser fluence was about $11-21 \mathrm{~J} / \mathrm{cm}^{2}$, and the repetition frequency was $5 \mathrm{~Hz}$. (111) Gadolinium Gallium Garnet (GGG) substrate was held at RT - $860{ }^{\circ} \mathrm{C}$. Two kinds of target, $\mathrm{Y}_{3.0} \mathrm{Fe}_{5.0} \mathrm{O}_{12.0}$ and $\mathrm{Y}_{3.0} \mathrm{Fe}_{7.8} \mathrm{O}_{16.2}$, were used. The deposition was carried out in the ambient gas of $\mathrm{O}_{2}$ with a low pressure of $2.7 \mathrm{~Pa}$. The relatively low pressure was selected on purpose to avoid the reduction of the droplet temperature due to collision with environmental neutral oxygen molecules of RT. The film morphology was inspected by the scanning electron microscopy (SEM) and the crystal structure was investigated by the X-ray diffraction (XRD) measurement. The film compositions were determined by the energy-dispersive X-ray analysis (EDX). For characterizing microwave properties, it is essential to evaluate the linewidth $\Delta H$ of the ferrimagnetic resonance (FMR). For the FMR measurement, an electron spin resonance (ESR) spectrometer (JEOL JES-RE1X) was used with $\mathrm{TE}_{011}$ cylindrical cavity operated at X-band $(9.3 \mathrm{GHz})$ with a field-modulation at a frequency of $100 \mathrm{kHz}$ and at RT. Two configurations were used with respect to the direction of applied magnetic field $\boldsymbol{H}$; magnetic field $\boldsymbol{H}$ applied parallel to the film plane and perpendicularly to the film plane. Hereafter, these two configurations are 
just denoted by "parallel" and "perpendicular", respectively. The microwave magnetic field is always parallel to the film plane because of the $\mathrm{TE}_{011}$ mode.

\section{Results and discussion}

In our preliminary experiment, YIG film growth was performed using PLA with ArF excimer laser. The FMR measurement shows that YIG films on (111) GGG substrates has a small FMR linewidth $\Delta H$, suggesting a low microwave loss in device applications [11]. The film growth was, however, found not to be mainly governed by droplets but by atoms, molecules, and/or small clusters because of the short wavelength of the laser $[3,12]$ and the limited laser fluence due to a large beam divergence.

For enhancing the YIG droplet ejection from the target, the second harmonic of Qswitched Nd-YAG laser was employed instead of ArF excimer laser. For estimating the ejected droplet density, SEM observation was performed for YIG films prepared at RT. The RT deposition allows us a rapid quenching of the molten droplets on the substrate surface without merging into the film, leading to an observation of all the droplets landing on the substrate. Figure 1 shows the SEM photographs of the YIG films prepared with a laser fluence of 10 $\mathrm{J} / \mathrm{cm}^{2}$ at RT on (111) GGG substrates placed on two positions. The target-substrate distances are (a) $35 \mathrm{~mm}$ and (b) $15 \mathrm{~mm}$. By decreasing the distance the number of droplets is found to be largely increased. As a result, the surface of the film prepared with the $15-\mathrm{mm}$ distance is covered by a large number of droplets with a micron-size. In addition, the shorter the distance is, the higher the droplet temperature on the substrate is [5]. Hereafter, for increasing the density of droplets, a short target-substrate distance of $15 \mathrm{~mm}$ was selected and the laser 
fluence is further increased up to $21 \mathrm{~J} / \mathrm{cm}^{2}$.

Under the preparation condition, the distance of $15 \mathrm{~mm}$ and the fluence of $21 \mathrm{~J} / \mathrm{cm}^{2}$, the substrate temperature was elevated up to $860{ }^{\circ} \mathrm{C}$ for enhancing the spreading (twodimensional growth) of the molten droplets and their crystal growth on (111) GGG substrate. Figure 2 shows the SEM photographs of the YIG films prepared at $860^{\circ} \mathrm{C}$ with a laser fluence of $21 \mathrm{~J} / \mathrm{cm}^{2}$ on (111) GGG substrates. Surface morphology was greatly improved by increasing the substrate temperature while there remained the traces of spreading molten droplets. Further increase of the substrate temperature will improve the surface morphology. In our apparatus, this substrate temperature is unfortunately near the highest limit.

YAG laser showed a disadvantage for stoichiometric film growth, though it is powerful for the efficient droplet production as mentioned above. Figure 3 shows the EDX composition of the films prepared using two targets of $\mathrm{Y}_{3} \mathrm{Fe}_{5} \mathrm{O}_{12}$ (open squares) and $\mathrm{Y}_{3} \mathrm{Fe}_{7.8} \mathrm{O}_{16.2}$ (closed circles) compositions with various substrate temperatures. Although for a preparation of the films using the $\mathrm{Y}_{3} \mathrm{Fe}_{5} \mathrm{O}_{12}$ target a small amount of ozone was added in order to increase the Fe content, there was almost no effect on the improvement. The Fe content is found to be increased by using the Fe-enriched target. Still there remained the Fe deficiency in films prepared at high substrate temperatures even if the Fe-enriched target was used. No large Fe deficiency was observed using ArF or $\mathrm{KrF}$ excimer lasers. So far, the reason for the Fe deficiency in the films deposited using YAG laser has not been clarified.

YIG films were grown using the above two targets with the target-substrate distance of $15 \mathrm{~mm}$ and a substrate temperature of $860{ }^{\circ} \mathrm{C}$. XRD patterns for the films using (a) $\mathrm{Y}_{3} \mathrm{Fe}_{5} \mathrm{O}_{12}$ and (b) $\mathrm{Y}_{3} \mathrm{Fe}_{7.8} \mathrm{O}_{16.2}$ targets are shown in Fig.4. YIG (444) diffractions adjacent to the GGG (444) diffraction were observed for the both films. No diffractions from the other crystal planes were observed in a wide range of $2 \theta$ scan for the both cases. These results suggest that [111] highly 
oriented YIG crystals were grown on the (111) GGG substrate by PLA using a large number of molten droplets. But for the film shown in Fig.4 (a) the YIG (444) diffraction line has a large width without $\mathrm{K}_{\alpha 1}-\mathrm{K}_{\alpha 2}$ splitting and that is not closed to the bulk diffraction position of $2 \theta=$ $51.083^{\circ}$, suggesting a poor crystal growth. The YIG (444) diffraction line for the film shown in Fig.4 (b) reveals a clear $\mathrm{K}_{\alpha 1}-\mathrm{K}_{\alpha 2}$ splitting and is approaching the bulk value, suggesting that an excellent crystal growth is achieved by the Fe-enriched target. The full width at half maximum of the rocking curve measurement is about 0.23 for the film prepared with $\mathrm{Y}_{3} \mathrm{Fe}_{7.8} \mathrm{O}_{16.2}$ targets, supporting the excellent crystal growth of YIG.

The FMR measurement was performed for these films. The result is shown in Fig. 5 for the films using (a) $\mathrm{Y}_{3} \mathrm{Fe}_{5} \mathrm{O}_{12}$ and (b) $\mathrm{Y}_{3} \mathrm{Fe}_{7.8} \mathrm{O}_{16.2}$ targets. The $\mathrm{YIG}$ film prepared using $\mathrm{Y}_{3} \mathrm{Fe}_{5} \mathrm{O}_{12}$ target shows a relatively large $\Delta H$ of 115 Oe while the film prepared using $\mathrm{Y}_{3} \mathrm{Fe}_{7.8} \mathrm{O}_{16.2}$ target shows a small $\Delta H$ of 7.5 Oe. The linewidth was defined as the separation between the neighboring peak-to-peak with the largest height. This value is quite small among films prepared by VPE techniques [13]. $\Delta \mathrm{H}$ for the YIG prepared by the conventional LPE is, however, reported to be no larger than 1 Oe [14]. For a further improvement of a microwave characteristic of YIG film, an increase of the substrate temperature is preferable. The increase of the substrate temperature is expected to give rise to a smooth film surface and an equilibrium crystal growth with a low defect density. Instead of the present YAG laser, an excimer laser with a rather high beam energy is also expected to allow us the LPE-like film growth from molten droplets without any film off-stoichiometry.

\section{Conclusions}


It was found that YIG films prepared by PLA at RT substrates have a large number of solidified droplets with various sizes. Highly oriented YIG crystals were grown on the (111) GGG substrate heated at $860^{\circ} \mathrm{C}$ using a large number of molten droplets, suggesting an LPE-like growth. This YIG film shows a small $\Delta \mathrm{H}$ of $7.5 \mathrm{Oe}$. This value is quite small among the films prepared by VPE techniques, while $\Delta \mathrm{H}$ for the YIG prepared by the conventional LPE is reported to be around 1 Oe.

\section{Acknowledgement}

We thank Professor M. Kumeda of Kanazawa University for stimulating discussions. We thank T. Kishi and K. Nagai of Kanazawa University for technical assistance. This work was partly supported by Grant-in-Aid for Scientific Research (B) from the Ministry of Education, Science, Sports and Culture of Japan (No.10450006) and by Foundation for Promotion of Material Science and Technology of Japan.

\section{Reference}

1. O. Eryu, K. Murakami, K. Masuda, A. Kasuya and Y. Nishina; Appl. Phys. Lett. 54, 2716 (1989).

2. O. Eryu, K. Murakami, K. Masuda, K. Shihoyama, T. Mochizuki; Jpn. J. Appl. Phys. 31, L86 (1992).

3. Y. Yonezawa, K. Segawa, S. Katayama, T. Minamikawa, A. Morimoto, and T. Shimizu; Jpn. J. Appl. Phys. 33, L1178 (1994). 
4. Y. Yonezawa, T. Minamikawa, K. Matsuda, K. Takezawa, A. Morimoto, and T. Shimizu; Appl. Surf. Sci., 127-129, 639 (1998).

5. A. Morimoto, S. Oguri, and T. Shimizu T. Minamikawa, and Y. Yonezawa (to be submitted elsewhere)

6. Y. K. Fetisov, P. Kabos, and C. E. Patton; IEEE Trans. Mag. 34, 259 (1998).

7. N. S. Chang and Y. Matsuo; Appl. Phys. Lett. 35, 352 (1979).

8. M. Dragoman and M. Catoiu; Appl. Phys. Lett. 54, 1472 (1989).

9. J. D. Adams, and S. N. Stitzer; Appl. Phys. Lett., 36, 485 (1980).

10. H. Kidoh, A. Morimoto, T. Shimizu; Appl. Phys. Lett., 59, 237 (1991).

11. C. Vittoria, and N. D. Wilsey; J. Appl. Phys. 45, 414 (1974).

12. G. Koren, A. Gupta, R. J. Baseman, M. I. Lutwyche, and R. B. Laibowitz, Appl. Phys. Lett. 55, 2450 (1989).

13. A. K. Srivastava, M. J. Hurben, M. A. Wittenauer, P. Kabos, R. Ramesh, P. C. Dorsey, and D. B. Chrisey; J. Appl. Phys. 85, 7838 (1999).

14. P. Kabos, C. E. Patton, G. Wiese, A. D. Sullins, E. S. Wright, and L. Chen; J. Appl. Phys., 80, 3962 (1996). 


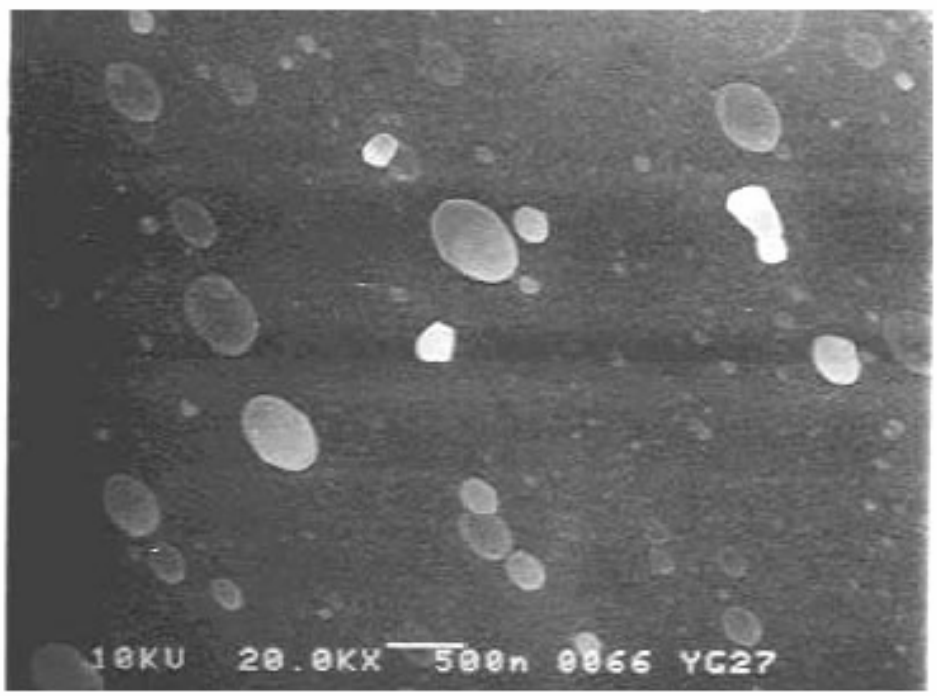

(a)

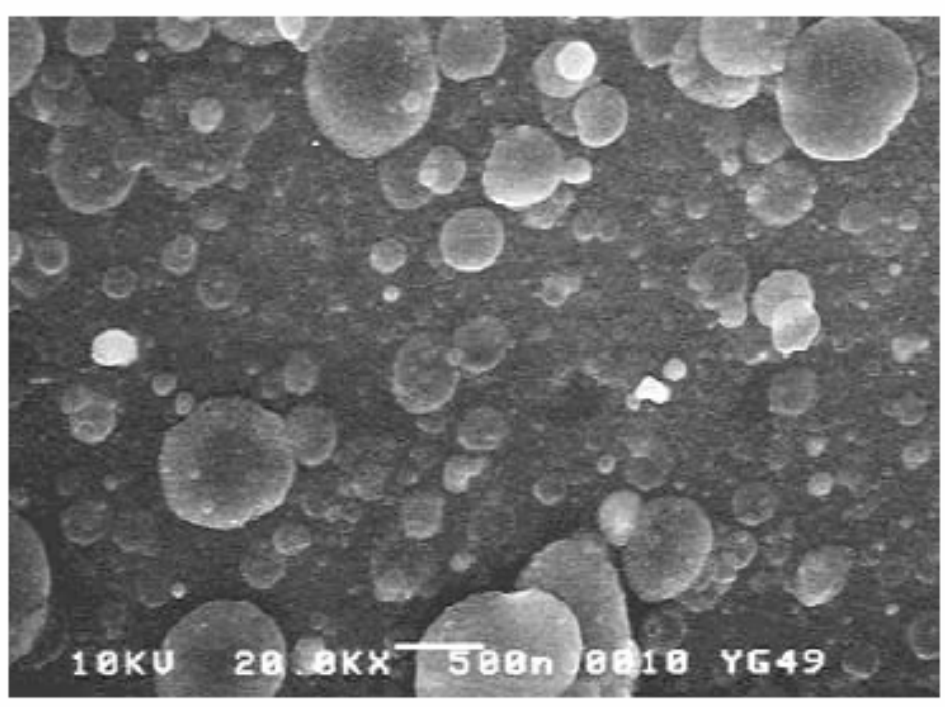

(b)

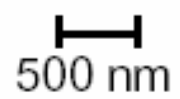

Figure 1 SEM photographs of the YIG films prepared using $\mathrm{Y}_{3} \mathrm{Fe}_{5} \mathrm{O}_{12}$ target with a laser fluence of $10 \mathrm{~J} / \mathrm{cm}^{2}$ at RT on (111) GGG substrates placed on two positions. The targetsubstrate distances are (a) $35 \mathrm{~mm}$ and (b) $15 \mathrm{~mm}$. 

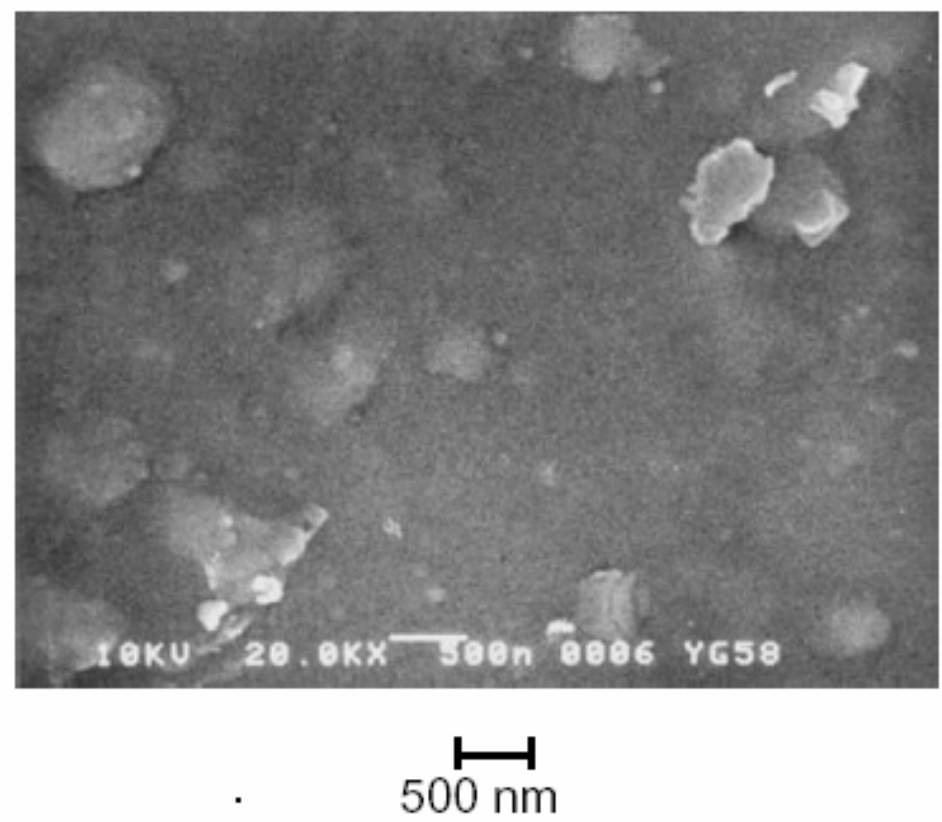

Figure 2 SEM photographs of the YIG films prepared at $860{ }^{\circ} \mathrm{C}$ with a laser fluence of $21 \mathrm{~J} / \mathrm{cm}^{2}$ and a distance of $15 \mathrm{~mm}$ on (111) GGG substrates using $\mathrm{Y}_{3} \mathrm{Fe}_{7.8} \mathrm{O}_{16.2}$ target.

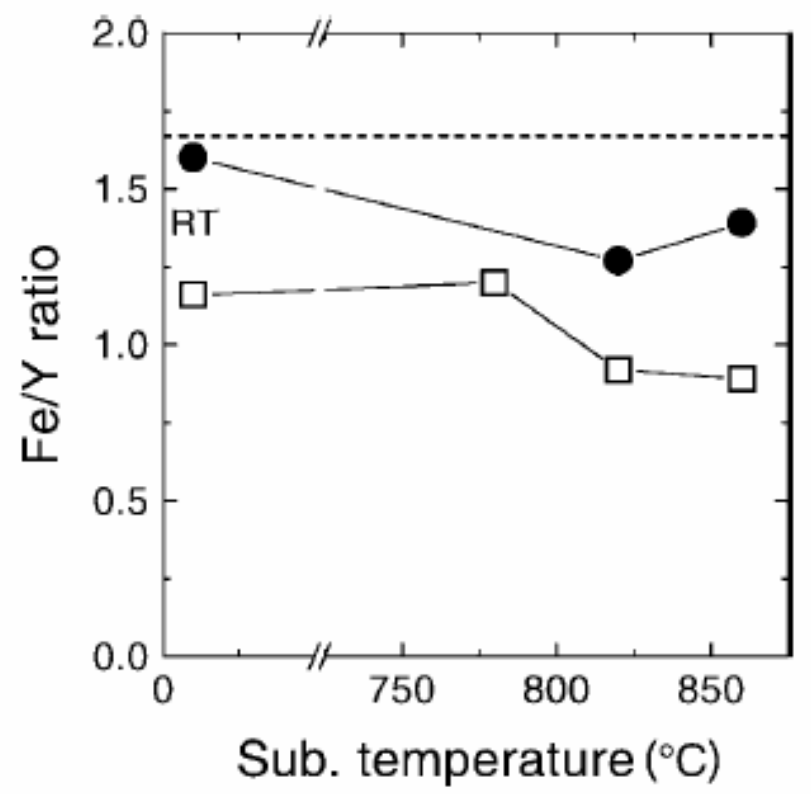

Figure 3 Composition of the films prepared using $\mathrm{Y}_{3} \mathrm{Fe}_{5} \mathrm{O}_{12}$ (open squares) and $\mathrm{Y}_{3} \mathrm{Fe}_{7.8} \mathrm{O}_{16.2}$ (solid circles) targets with various substrate temperatures. The horizontal broken line represents the stoichiometric composition of 5/3. 


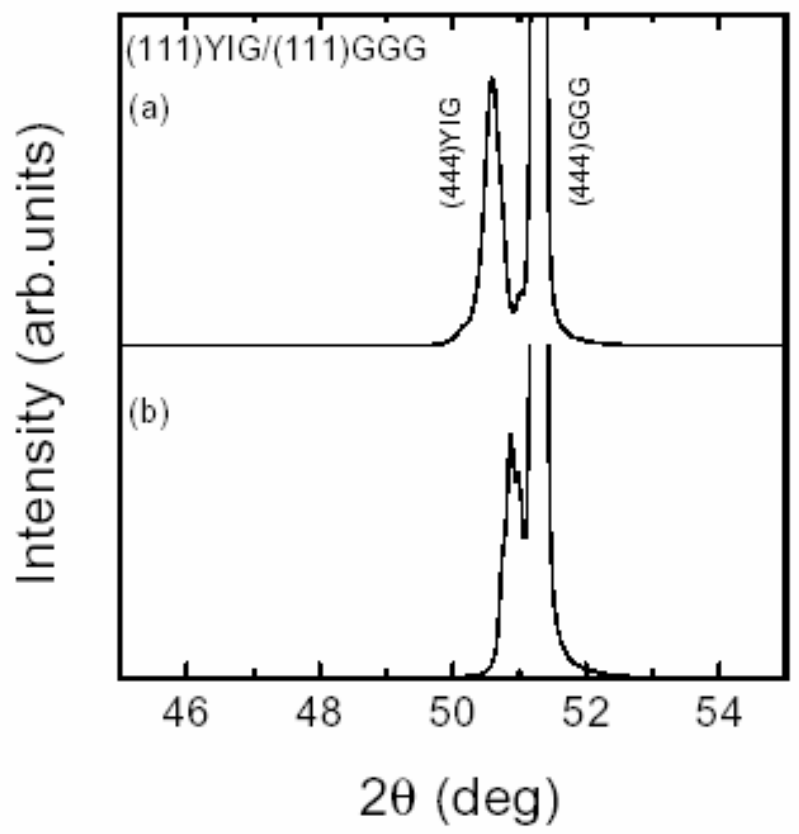

Figure $4 \mathrm{XRD}$ patterns for the films prepared at $860{ }^{\circ} \mathrm{C}$ with a laser fluence of $21 \mathrm{~J} / \mathrm{cm}^{2}$ and a distance of $15 \mathrm{~mm}$ using (a) $\mathrm{Y}_{3} \mathrm{Fe}_{5} \mathrm{O}_{12}$ and (b) $\mathrm{Y}_{3} \mathrm{Fe}_{7.8} \mathrm{O}_{16.2}$ targets. 

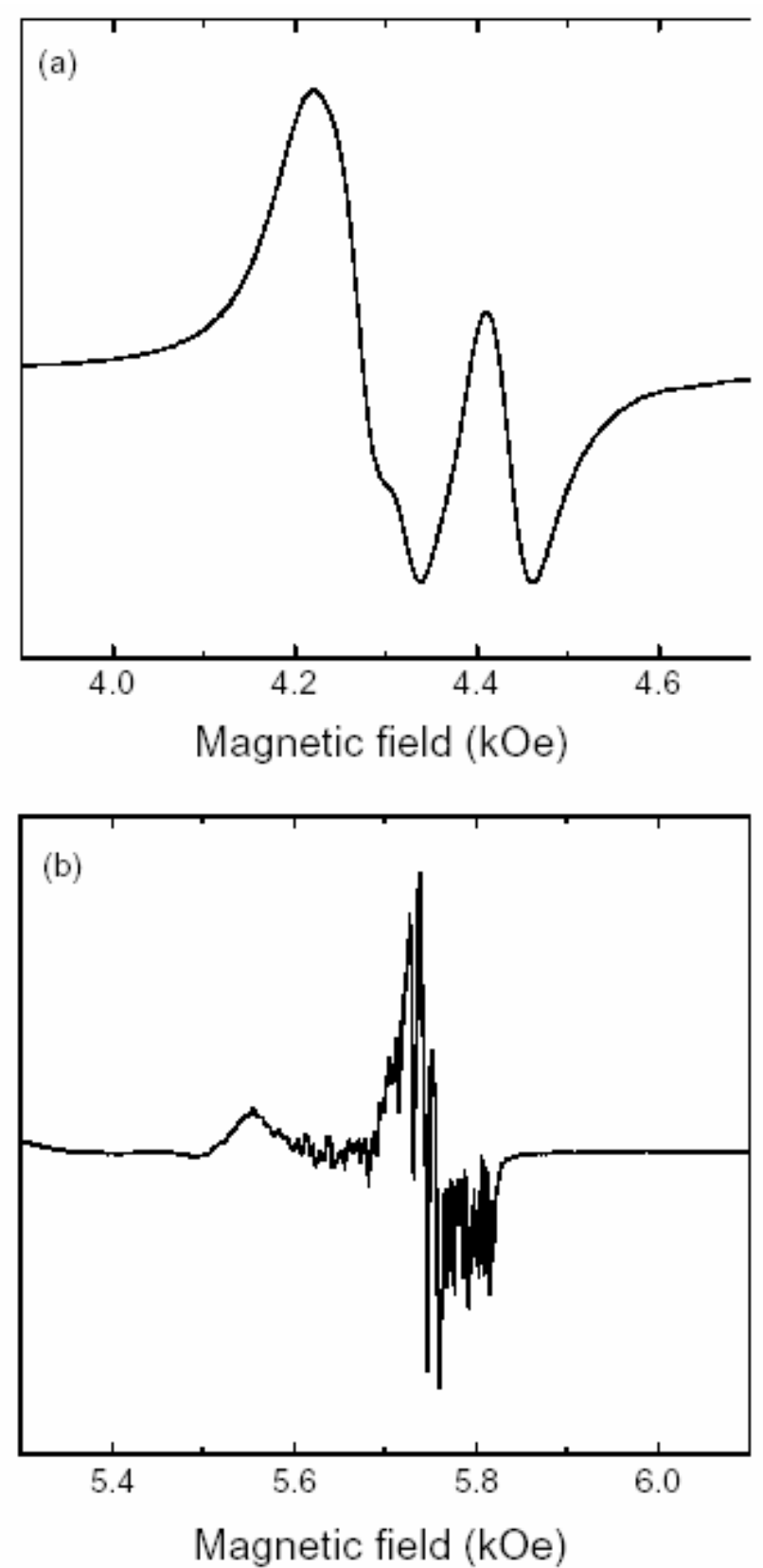

Figure 5 FMR spectra for the films prepared at $860{ }^{\circ} \mathrm{C}$ with a laser fluence of $21 \mathrm{~J} / \mathrm{cm}^{2}$ and a distance of $15 \mathrm{~mm}$ using (a) $\mathrm{Y}_{3} \mathrm{Fe}_{5} \mathrm{O}_{12}$ and (b) $\mathrm{Y}_{3} \mathrm{Fe}_{7.8} \mathrm{O}_{16.2}$ targets. The configuration is "perpendicular". 
13,500 keystrokes (including spaces) $+2,100$ keystrokes x 5 figures

$=24,000$ keystrokes $<25,000$ keystrokes 